\title{
Radial Solution of the S-Wave Schrodinger Equation with Kratzer Plus Modified Deng-Fan Potential Under the Framework of Nikifarov-Uvarov Method
}

\author{
Louis Hitler ${ }^{1,2}$, Benedict Iserom Ita ${ }^{1}$, Ozioma Udochuku Akakuru', Thomas Odey Magu ${ }^{1}$, \\ Innocent Joseph ${ }^{3}$, Pigweh Amos Isa ${ }^{3, *}$ \\ ${ }^{1}$ Physical/Theoretical Chemistry Research Group, Department of Pure and Applied Chemistry, University oF Calabar, Calabar, Nigeria \\ ${ }^{2}$ CAS Key Laboratory For Nanosystem and Hierarchical Fabrication, CAS Centre For Excellence In Nanoscience, National Centre for \\ Nanoscience and Technology, University of Chinese Academy of Science, Beijing, China \\ ${ }^{3}$ Department of Chemistry, Modibbo Adama University of Technology, Yola, Nigeria
}

Email address:

louismuzong@gmail.com (P. A. Isa), amospigweh@gmail.com (P. A. Isa)

${ }^{*}$ Corresponding author

To cite this article:

Louis Hitler, Benedict Iserom Ita, Ozioma Udochuku Akakuru, Thomas Odey Magu, Innocent Joseph, Pigweh Amos Isa. Radial Solution of the S-Wave Schrodinger Equation with Kratzer Plus Modified Deng-Fan Potential Under the Framework of Nikifarov-Uvarov Method. International Journal of Applied Mathematics and Theoretical Physics. Vol. 3, No. 4, 2017, pp. 97-100. doi: 10.11648/j.ijamtp.20170304.14

Received: September 14, 2017; Accepted: October 18, 2017; Published: December 21, 2017

\begin{abstract}
The solutions of the Schrodinger equation with Kratzer plus Modified Deng-Fan potential have been obtained using the parametric Nikiforov-Uvarov (NU) method which is based on the solutions of general second-order linear differential equations with special functions. The bound state energy eigenvalues and the corresponding un-normalized eigen functions are obtained in terms of Jacobi polynomials. Also special cases of the potential have been considered and their energyeigen values obtained.
\end{abstract}

Keywords: Schrodinger, Kratzer, Deng-Fan, Eigen Energy

\section{Introduction}

The exact analytic solutions of nonrelativistic and relativistic wave equations are only possible for certain potentials of physical interest. It is well known and understood that the analytical exact solutions of these wave equations are only possible in a few cases such as the harmonic oscillator, Coulomb, pseudoharmonic potentials and others [1]. For $l \neq 0$ approximation techniques have to be employed to deal with the centrifugal term like the Pekeris approximation $[2,3]$ and the approximated scheme suggested by Greene and Aldrich. Some of these exponential-type potentials include the Hulthen potential [4], the Morse potential [5], the Woods-Saxon potential [3], the Kratzer-type and pseudoharmonic potentials [3, 2], the Rosen-Morse-type potentials [6], the Manning-Rosen potential [3]. Recently our groups have attempted to study the bound state solutions of Klein-Gordon, Dirac and Schrodinger equations using combined or mixed potentials. Some of which includes Woods-Saxon plus Attractive Inversely Quadratic potential (WSAIQP) [7], Manning-Rosen plus a class of Yukawa potential (MRCYP) [8], generalized wood-saxon plus Mietype potential (GWSMP) [9], Kratzer plus Reduced Pseudoharmonic Oscillator potential (KRPHOP)[10, 2], Inversely Quadratic Yukawa plus attractive radial potentials (IQYARP) [11], Modified Echart Plus Inverse Square Molecular Potentials (MEISP) [12]. The Deng-Fan molecular potential is a simple modified Morse potential called the generalized Morse potential, which was proposed by Deng and Fan in 1957 [13], in an attempt to find a more suitable diatomic potential to describe the vibrational spectrum [14]. Although, this potential is qualitatively similar to the Morse potential but it has correct asymptotic behavior as the internuclear distance approaches to zero [15]. This potential has been used to describe diatomic molecular energy spectra and electromagnetic transitions, and it is an ideal internuclear potential in diatomic molecules with the same molecular 
behavior [15]. However, not much has been achieved in the area of solving the relativistic Schrodinger equation with Kratzer plus Modified Deng-Fan potential (KMDFP) using Nikiforov-Uvarov (NU) method which is the purpose of this paper. This study is organized as follows: Section 2 contains the overview of the Nikiforov-Uvarov method. In Section 3, the Schrodinger equation with Kratzer plus Modified DengFan potentials (KMDFP) is solved by using the NikiforovUvarov method. The relativistic energy equations and the corresponding unnormalized wave functions are obtained and finally, the conclusion is given in Section 4.

\section{Review of Parametric Nikifarov-Uvarov Method}

The NU method is based on the solutions of a generalized second order linear differential equation with special orthogonal functions. The Nikiforov-Uvarov method has been successfully applied to relativistic and nonrelativistic quantum mechanical problems and other field of studies as well [6-9]. The hypergeometric NU method has shown its power in calculating the exact energy levels of all bound states for some solvable quantum systems.

$$
\Psi_{\mathrm{n}}^{\prime \prime}(\mathrm{s})+\frac{\tilde{\tau}(s)}{\sigma(s)} \Psi_{\mathrm{n}}^{\prime}(\mathrm{s})+\frac{\bar{\sigma}(s)}{\sigma^{2}(s)} \Psi_{\mathrm{n}}(\mathrm{s})=0
$$

Where $\sigma(\mathrm{s})$ and $\bar{\sigma}(\mathrm{s})$ are polynomials at most second degree and $\tilde{\tau}(\mathrm{s})$ is first degree polynomials. The parametric generalization of the N-U method is given by the generalized hypergeometric-type equation

$$
\Psi^{\prime \prime}(s)+\frac{c_{1}-c_{2} s}{s\left(1-c_{3} s\right)} \Psi^{\prime}(s)+\frac{1}{s^{2}\left(1-c_{3} s\right)^{2}}\left[-\epsilon_{1} s^{2}+\epsilon_{2} s-\epsilon_{3}\right] \Psi(s)=0
$$

Thus eqn. (1) can be solved by comparing it with equation (2) and the following polynomials are obtained

$$
\begin{gathered}
\tilde{\tau}(s)=\left(c_{1}-c_{2} s\right), \sigma(s)=s\left(1-c_{3} s\right), \bar{\sigma}(s)=-\epsilon_{1} s^{2}+ \\
\epsilon_{2} s-\epsilon_{3}
\end{gathered}
$$

The parameters obtainable from equation (3) serve as important tools to finding the energy eigenvalue and eigenfunctions. They satisfy the following sets of equation respectively

$$
\begin{gathered}
\mathrm{c}_{2} \mathrm{n}-(2 \mathrm{n}+1) \mathrm{c}_{5}+(2 \mathrm{n}+1)\left(\sqrt{c_{9}}+\mathrm{c}_{3} \sqrt{c_{8}}\right)+\mathrm{n}(\mathrm{n}-1) c_{3}+c_{7}+2 c_{3} c_{8} \\
+2 \sqrt{c_{8} c_{9}}=0 \\
\left(\mathrm{c}_{2}-\mathrm{c}_{3}\right) \mathrm{n}+c_{3} \mathrm{n}^{2}-(2 \mathrm{n}+1) c_{5}+(2 \mathrm{n}+1)\left(\sqrt{c_{9}}+\mathrm{c}_{3} \sqrt{c_{8}}\right)+c_{7}+ \\
2 c_{3} c_{8}+2 \sqrt{c_{8} c_{9}}=0
\end{gathered}
$$

While the wave function is given as

$$
\Psi_{n}(s)=N_{n, l} S^{c_{12}}\left(1-c_{3} s\right)^{-c_{12}-\frac{c_{13}}{c_{3}} P_{n}}{ }_{\left.2 c_{3} s\right)}^{\left(c_{10}-1, \frac{c_{11}}{c_{3}}-c_{10}-1\right)}(1-
$$

Where

$$
c_{4}=\frac{1}{2}\left(1-c_{1}\right), c_{5}=\frac{1}{2}\left(c_{2}-2 c_{3}\right),
$$

$$
\begin{aligned}
& c_{6}=c_{5}{ }^{2}+\epsilon_{1}, c_{7}=2 c_{4} c_{5}-\epsilon_{2}, \\
& c_{8}=c_{4}{ }^{2}+\epsilon_{3}, \\
& c_{9}=c_{3} c_{7}+c_{3}{ }^{2} c_{8}+c_{6}, \\
& c_{10}=c_{1}+2 c_{4}+2 \sqrt{c_{8}}, \\
& c_{11}=c_{2}-2 c_{5}+2\left(\sqrt{c_{9}}+\mathrm{c} 3 \sqrt{c_{8}}\right) \\
& c_{12}=c_{4}+\sqrt{c_{8}}, \\
& c_{13}=c_{5}-\left(\sqrt{c_{9}}+\mathrm{c} 3 \sqrt{c_{8}}\right)
\end{aligned}
$$

and $P_{n}$ is the orthogonal polynomials.

$$
\begin{aligned}
& \text { Given that } P_{n}^{(\alpha, \beta)}= \\
& \sum_{r=0}^{n} \frac{\Gamma(n+\alpha+1) \Gamma(n+\beta+1)}{\Gamma(\alpha+r+1) \Gamma(n+\beta-r+1)(n-r) ! r !}\left(\frac{x-1}{2}\right)^{r}\left(\frac{x+1}{2}\right)^{n-r} \text { (8) }
\end{aligned}
$$

This can also be expressed in terms of the Rodriguez's formula

$$
\begin{aligned}
& P_{n}^{(\alpha, \beta)}(x)=\frac{1}{2^{n} n !}(x-1)^{-\alpha}(x \\
& +1)^{-\beta}\left(\frac{d}{d x}\right)^{n}\left((x-1)^{n+\alpha}(x+1)^{n+\beta}\right)
\end{aligned}
$$

\section{Bound State Solution of the Schrodinger Equation for Kmdfp}

The Schrodinger Equation with vector $V(r)$, potential in atomic units $(\hbar=\mathrm{c}=1)$ is given as

$$
\frac{d^{2} R(r)}{d r^{2}}+\frac{2 \mu}{\hbar^{2}}[(E-V(r))] R(r)=0
$$

Where $E, V(r), h$ are total energy, potential and planck's constant respectively.

The Modified Deng-Fan Potential [13], is given as

$$
V(r)=-\left(A_{1}+\frac{A_{2}}{(1-s)}+\frac{A_{3}}{(1-s)^{2}}\right)
$$

Where $A_{1}, A_{2}$ and $A_{3}$ are constant that depends on the dissociation energy.

The Kratzer Potential [17], is given as

$$
V(r)=\frac{V_{1} e^{-\alpha r}}{1-e^{-\alpha r}}
$$

Where, screening parameter $\alpha$ determines the range of the potential, and $V_{1}$, is the coupling parameter describing the depth of the potential well. In general term, the hyperbolic functions are defined as

$$
\begin{gathered}
\operatorname{Sinh}_{q}(r)=\frac{1}{\operatorname{Cosech}_{q}(r)}=\frac{e^{r}-q e^{-r}}{2}, \operatorname{Cosh}_{q}(r)= \\
\frac{e^{r}+q e^{-r}}{2}, \operatorname{Coth}_{q}(r)=\frac{\operatorname{Cosh}_{q}(r)}{\operatorname{Sinh}_{q}(r)}
\end{gathered}
$$

Making the transformation $s=e^{-2 \alpha r}$ the sum of the potentials (KMDFP) in equations (10) and (11) becomes 


$$
V(r)=-\left(A_{1}+\frac{A_{2}}{(1-s)}+\frac{A_{3}}{(1-s)^{2}}+\frac{V_{1} s}{(1-s)}\right)
$$

Substitute eq. (12) into the Schrodinger equation given in equation (9) we have

$$
\begin{aligned}
& \frac{d^{2} R(r)}{d r^{2}}+ \\
& \frac{2 \mu}{\hbar^{2}}\left[\left(E+\left(\left(A_{1}+\frac{A_{2}}{(1-s)}+\frac{A_{3}}{(1-s)^{2}}+\frac{V_{1} s}{(1-s)}\right)\right)\right] R(r)=0\right.
\end{aligned}
$$

Applying the Pekeris-like approximation [16] given as $\frac{1}{r^{2}}=\frac{4 \alpha^{2}}{(1-s)^{2}}$ to eq. (14) enable us completely solve eq. (9).

Again, applying the transformation $s=e^{-2 \alpha r}$ to get the form that NU method is applicable, equation (9) gives a generalized hypergeometric-type equation as

$$
\begin{array}{r}
\frac{d^{2} R(s)}{d s^{2}}+\frac{(1-s)}{(1-s) s} \frac{d R(s)}{d s}+\frac{1}{(1-)^{2} s^{2}}\left[\left(2 \beta^{2}+B+K\right) s^{2}+\right. \\
\left.\left(P-K-2 B-4 \beta^{2}\right) s+\left(2 \beta^{2}+H+B+P\right)\right] R(s)=0(15)
\end{array}
$$

Where

$$
\begin{gathered}
\beta^{2}=\left(\frac{\mu E}{4 \alpha^{2} \hbar^{2}}\right), B=\left(\frac{\mu}{2 \alpha^{2} \hbar^{2}}\right) A_{1}, k=\left(\frac{\mu}{2 \alpha^{2} \hbar^{2}}\right) V_{1}, P= \\
\left(\frac{\mu}{2 \alpha^{2} \hbar^{2}}\right) A_{2}, H=\left(\frac{\mu}{2 \alpha^{2} \hbar^{2}}\right) A_{3}
\end{gathered}
$$

Comparing equation (14) with equation (2) yields the following parameters

$$
\begin{gathered}
c_{1}=c_{2}=c_{3}=1, c_{4}=0, \\
c_{5}=-\frac{1}{2}, c_{6}=\frac{1}{4}+2 \beta^{2}+B+K, \\
\left\{\left[\frac{-\left(2\left(\frac{\mu}{2 \alpha^{2} \hbar^{2}}\right) A_{2}+\left(\frac{\mu}{2 \alpha^{2} \hbar^{2}}\right) A_{1}+\left(\frac{\mu}{2 \alpha^{2} \hbar^{2}}\right) A_{3}\right)}{(2 n+1)+2 \sqrt{\frac{1}{4}+\left(\frac{1}{2 \alpha^{2}}\right.}}\right.\right. \\
\text { culate the radial wave function of } \\
\rho(s)=s^{u}(1-q s)^{v} \\
2 \beta^{2}-R-B+\lambda, \text { and } v=2 \sqrt{\frac{1}{4}-B-B} \\
X_{n}(s)=p_{n}^{(u, v)}(1-2 s), \\
\varphi(s)=s^{u / 2}(1-s)^{1+v / 2}
\end{gathered}
$$$$
E=\frac{4 \alpha^{2} \hbar^{2}}{\mu}\left\{\left[\frac{-\left(2\left(\frac{\mu}{2 \alpha^{2} \hbar^{2}}\right) A_{2}+\left(\frac{\mu}{2 \alpha^{2} \hbar^{2}}\right) A_{1}+\left(\frac{\mu}{2 \alpha^{2} \hbar^{2}}\right) A_{3}\right)-\left(n^{2}+n-\frac{1}{2}\right)-(2 n+1) \sqrt{\frac{1}{4}+\left(\frac{\mu}{2 \alpha^{2} \hbar^{2}}\right) A_{3}}}{(2 n+1)+2 \sqrt{\frac{1}{4}+\left(\frac{\mu}{2 \alpha^{2} \hbar^{2}}\right) A_{3}}}\right]\right\}-\left(\left(\frac{\mu}{2 \alpha^{2} \hbar^{2}}\right) A_{2}+\left(\frac{\mu}{2 \alpha^{2} \hbar^{2}}\right) A_{1}+\left(\frac{\mu}{2 \alpha^{2} \hbar^{2}}\right) A_{3}\right)(19
$$

We now calculate the radial wave function of the KMDFP as follows

Where $u=2 \beta^{2}-R-B+\lambda$, and $v=2 \sqrt{\frac{1}{4}-B-H-P+\lambda}$

Using equation (16) we get the function $\chi(s)$ as

$$
\chi(s)=P_{n}^{(U, V)}(1-2 s),
$$

Where $P_{n}^{(U, V)}$ are Jacobi polynomials

Lastly,

$$
\varphi(s)=s^{c_{12}}\left(1-c_{3} s\right)^{-c_{12}-\frac{c_{13}}{c_{3}}},
$$

And using equation (16) we get

$$
\varphi(s)=s^{U / 2}(1-s)^{V-1 / 2},
$$

$$
\begin{aligned}
& c_{7}=-4 \beta^{2}-2 B+P-K, \\
& c_{8}=2 \beta^{2}+H+B+P, c_{9}=\frac{1}{4}+H, \\
& c_{10}=1+2 \sqrt{2 \beta^{2}+H+B+P}, \\
& c_{11}=2+2\left(\sqrt{\frac{1}{4}+H}+\sqrt{2 \beta^{2}+H+B+P}\right), \\
& c_{12}=\sqrt{2 \beta^{2}+H+B+P}, \\
& c_{13}=-\frac{1}{2}-\left(\sqrt{\frac{1}{4}+H}+\sqrt{2 \beta^{2}+H+B+P}\right), \\
& \varepsilon_{1}=2 \beta^{2}+B+K, \varepsilon_{2}=4 \beta^{2}+2 B-P+K, \\
& \varepsilon_{3}=2 \beta^{2}+H+B+P
\end{aligned}
$$

Now using equations (6), (16) and (17) we obtain the energy eigen spectrum of the KMDFP as

$$
\beta^{2}=\left[\frac{-(2 P+B+H)-\left(n^{2}+n-\frac{1}{2}\right)-(2 n+1) \sqrt{\frac{1}{4}+H}}{(2 n+1)+2 \sqrt{\frac{1}{4}+H}}\right]^{2}-(B+P+H)(
$$

The above equation can be solved explicitly with the substitution of values, equation (18) yields the explicit energy eigen spectrum of KMDFP as
We then obtain the radial wave function from the equation

$$
R_{n}(s)=N_{n} \varphi(s) \chi_{n}(s),
$$

As

$$
R_{n}(s)=N_{n} s^{U / 2}(1-s)^{(V-1) / 2} P_{n}^{(U, V)}(1-2 s),
$$

Where $n$ is a positive integer and $N_{n}$ is the normalization constant

\section{Discussion}

We consider the following cases from equation (19)

CASE I: If we choose $A_{1}=A_{2}=A_{3}=0$ then the energy eigen values of the Kratzer potential becomes

$$
E=\frac{4 \alpha^{2} \hbar^{2}}{\mu}\left\{\left[\frac{\left(n^{2}+n-\frac{1}{2}\right)-(2 n+1) \frac{1}{2}}{(2 n+1)+1}\right]\right\}
$$

CASE II: If we choose $V_{1}=0$ then the energy eigen values ofthe Deng-Fenf potential becomes 


$$
E=\frac{4 \alpha^{2} \hbar^{2}}{\mu}\left\{\left[\frac{-\left(2\left(\frac{\mu}{2 \alpha^{2} \hbar^{2}}\right) A_{2}+\left(\frac{\mu}{2 \alpha^{2} \hbar^{2}}\right) A_{1}+\left(\frac{\mu}{2 \alpha^{2} \hbar^{2}}\right) A_{3}\right)-\left(n^{2}+n-\frac{1}{2}\right)-(2 n+1) \sqrt{\frac{1}{4}+\left(\frac{\mu}{2 \alpha^{2} \hbar^{2}}\right) A_{3}}}{(2 n+1)+2 \sqrt{\frac{1}{4}+\left(\frac{\mu}{2 \alpha^{2} \hbar^{2}}\right) A_{3}}}\right]\right\}-\left(\left(\frac{\mu}{2 \alpha^{2} \hbar^{2}}\right) A_{2}+\left(\frac{\mu}{2 \alpha^{2} \hbar^{2}}\right) A_{1}+\left(\frac{\mu}{2 \alpha^{2} \hbar^{2}}\right) A_{3}\right)
$$

CASE III: when $A 1=A 2=A 3=A$, then equation (19) becomes

$$
E=\frac{4 \alpha^{2} \hbar^{2}}{\mu}\left\{\left[\frac{-\left(3 A\left(\frac{\mu}{2 \alpha^{2} \hbar^{2}}\right)\right)-\left(n^{2}+n-\frac{1}{2}\right)-(2 n+1) \sqrt{\frac{1}{4}+\left(\frac{\mu}{2 \alpha^{2} \hbar^{2}}\right) A}}{(2 n+1)+2 \sqrt{\frac{1}{4}+\left(\frac{\mu}{2 \alpha^{2} \hbar^{2}}\right) A}}\right]\right\}-\left(3 A\left(\frac{\mu}{2 \alpha^{2} \hbar^{2}}\right)\right)
$$

\section{Conclusion}

The Schrodinger equation for the sum of KP and MDFP referred to as KMDFP has been solved and solutions obtained using the Nikiforov-Uvarov (NU) method. The corresponding unnormalized eigen functions are evaluated in terms of Jacobi polynomials. Interestingly, the Klein-Gordon and Dirac equation with the arbitrary angular momentum values for this potential can be solved by this method. The resulting eigen energy equations can be used to study the spectroscopy of some selected diatomic atoms and molecules.

\section{References}

[1] Sameer M. Ikhdair (2011): Journal of Mathematical Physics 52, 052303 (2011); doi: http://dx.doi.org/10.1063/1.3583553

[2] Louis H., Ita B. I., Nelson N. A., I. Joseph., Amos P. I and Magu T. O...International Research Journal Of Pure and Applied Physics. Vol. 5, No. 3, pp 27-32, 2017.

[3] Sameer M. Ikhdairand Ramazan(2008). 10.2478/s11534-0080018-0.

[4] Sameer M. Ikhdair and Ramazan Sever (2009): Int. J. Mod. Phys. C 20 (1) (2009).

[5] Philip .M. Morse (1929): Phys. Rev. 34, 57 - Published 1 July 1929.

[6] N. Rosen and Philip M. Morse (1932 Phys. Rev. 42, 210 -
Published 15 October 1932.

[7] B. I. Ita, H. Louis, T. O. Magu and N. A. Nzeata-Ibe (2017. WSN 74 (2017) 280-287.

[8] H. Louis, B. I. Ita, T. O. Magu and N. A Nzeata-Ibe (2017. World scientific news, WSN 70(2) (2017) 312-319.

[9] B. I. Ita, B. E. Nyong., H. Louis, T. O. Magu (2017): J. Chem. Soc. Nigeria, Vol. 41, No. 2, pp21-26 [2017].

[10] H. Louis ${ }^{*}$, B. I. Ita., B. E. Nyong., T. O. Magu, S. Barka and N. A. Nzeata-Ibe (2016): Journal of Nigerian Association of Mathematical Physics vol.36, No. 2, (July, 2016) pp.193-198.

[11] B. I. Ita, B. E. Nyong, N. O. Alobi, H. Louis and T. O. Magu (2016):. Equatorial Journal of Computational and Theoretical Sciemces, Volume 1, Issue 1, (2016), pp. 55-64.

[12] B. I. Ita, A. I. Ikeuba, H. Louis and P. Tchoua (2015. Journal of Theoretical Physics and Cryptography. IJTPC, Vol. 10, December, 2015.www.IJTPC.org

[13] Z. H. Deng and Y. P. Fan, Shandong Univ. J. 7, 162 (1957).

[14] S. H. Dong, Factorization Method in Quantum Mechanics (Fundamental Theoriesin Physics, 150, Springer, Netherlands, 2007).

[15] L. H. Zhang, X. P. Li and C. S. Jia, Int. J. Quant. Chem. 111, $1870(2010)$.

[16] Louis H., Nzeata-Ibe N. A., Ita B. I., Joseph I., Isa P. A., Magu T. O: World Journal of Applied Physics, Vol. 2, N0. 5, 2017 , pp. 77-84. doi: 10.11648/j.wjap.20170205.13

[17] Yan-Fu Cheng and Tong-Qing Dai (2007. Phys. Scr. 75274. 